\title{
Portable Knowledge Sources for Machine Translation
}

\author{
Koichi Takeda \\ Tokyo Research Laboratory, IBM Research \\ 1623-14 Shimotsuruma, Yamato, Kanagawa 242, Japan \\ Phone: 81-462-73-4569, 81-462-73-7413 (FAX) \\ takeda@trl.vnet.ibm.com
}

\begin{abstract}
In this paper, we describe the acquisition and organization of knowledge sources for machine translation (M'l) systems. It has been pointed out by many users that one of the most annoying things about $\mathrm{M}^{\prime} \mathrm{l}^{\mathrm{s}}$ systems is the repeated occurrence of identical errors in word sense and attachment disambiguation. We show the limitations of a conventional user-dictionary method and explain how our approach solves the problem.
\end{abstract}

\section{Introduction}

In the last decale, more and nore commercial machine translation (MT) systems have become available for a wide variety of language pairs. An M'l' system is a very handy tool, but one quickly finds out that it makes the same errors over and over again even if a user dictionary is carefully maintuined. There are several reasoms for such repeated errors.

1. Commercial MT systems are not built in accordance with a powerful lexical semantic formalism. The user dictionary alone cannot disambiguate word senses and phrasal attachments satisfactorily.

2. MT systems cannot hardle the rlomain and contrxt dependency of word sense, phrasal attachment, and word selection.

3. In a shared environment, each user has a different user dictionary, and must therefore redundantly correct the same errors as all the other users.

A powerful lexical semantic approach ${ }^{[B]}$ could give more accurate translation, but it might be too much to ask users to develop their dictionaries within that formalism. The simple structure of a user dictionary also restricts the learning ability of MT systems during the post-editing process. The second of the above reasons has motivated recent example-based and case-based matchine translation research $[9,6,10]$. However, a method for finding the best-matching cases in a case base, where cases (or examples) are collected from difterent domains or contexts, has not been studied well. Nor is it known whether considering the frequency of cases gives a better result. The third reason is rarely rliscussed, but it is not desirable simply to share a single user dictionary, since the dictionary may become inconsistent by reflect ing multiple users' updates. Mckoy ${ }^{[5]}$ discussed word semse disambiguation using multiple knowledge sources, but her method is still dictionary-based.

Some of the rommercial systems for human-aided translation, such as the Translation Manager/2 ${ }^{[2]}$, can provide the user with more flexible access to multiple clictionaries and the translation memony (a repository of pairs of source and target sentences). 'This organization of knowledge could be quite useful for selecting correct translations of words, but the types of knowled ge available from the dictionaries and translation memo. ries are rather limited, and are certainly not enough for resolving structural ambignities in sentences.

In this paper, we propose Portable Knowledge Sources (PKSs) for machine translation. $\Lambda$ PKS consists of preference information on word sense, phrasal attachment, and word selection for translation. It is acquired through user interaction in the post-editing process, and is stored with the docmment being translated. When translating a document by using in M'T system, a user can spocify a list of already-translated documents, and the systen will make use of the PKSs included in the specilied documents. Wo show how such a collection of PKSs is organized, used for translation, and integrated into a user dictionary, and how the problem stated above can be solved by using PKSs.

\section{Portable Knowledge Sources}

A Portalble Knowledge Sonree (PKS) consists of preference information on three kinds of ambiguity:

1. Word sonse

2. Ply rasial attachement

3. Word selection

'The preference information is acquired from the user through postediting or interactive translation $[1,1]$, and is paired with the document that the user is working on. That is, a PKS is stored and managed together with the document for which it is created.

I, PK1, PK2, and PK3 be PKSs for the respective types of ambiguity mentioned above. The following is an example of word sense ambiguity:

Deletre the line. 
The word "line" could be (1) a single row of letters, (2) a geometric mark, (3) a hardware wire, and so on, for each of which a different translation is usually required in a target language. When the user specifies that a particular occurrence of the word "line" in a document D means a single row, the PKS

\section{(PK1 ("line" (cat $n$ )) (sense 1))}

is created, and is stored with $D$.

An example of phrasal attachment ambiguity is as follows:

Order the publication through the IBM branch serving your locality.

The present participle phrase can be either altached to the main verb "order," or to the noun "branch." If the user specifies that it modifies the noun as a postnominal adjective phrase (ADJP), the PKS

(PK2 ("serve" (cat v) (form prsprt)) ADJP ("branch" (cat $n))$ )

is created. ${ }^{1}$ The preference of prepositional phrase attachment is also represented by PK2.

Finally, an example of word selection ambiguity is (1) “メモリー・チップ" and (2) “販憶素子” for the compound noun "memory chip", where the first translation can often be found in PC documents, while the second one, which has the same meaning, is typically used in textbooks. When the user specifies that the second onc should be used, the PKS

(PK3 ("memory chip" (cat $n$ )) ("距隐素子" (cat $n)$ ))

is created. If word sense is to be included in the definition of word selection, such that the word W1 is used in sense $\mathrm{S}$ and should be translated by the word $W 2$, it is separately represented by (PK1 WI S) and (PK3 W1 W2).

Each PKS collected through user interaction has an age, based on the time and date of its creation. The younger the PKS, the stronger its preferability in one document, since it could have been used to overrule the preceding PKSs. Note that the age of a PKS is valid only among other PKSs in the same set. T'wo sets of PKSs are not comparable if they are paired with different documents.

\section{Organizing Portable Knowledge Sources}

Once the user has translated several documents, the sets of PKSs paired with them begin helping the M'I system to resolve the three kinds of a mbiguity described

\footnotetext{
${ }^{1}$ The representation of the PKS can vary depending on the MT system that uses the PKS. For example, the modifier and modified phrases can be represented by syntactic structures; word senses and semantic case relations can be associnted; and so on.
}
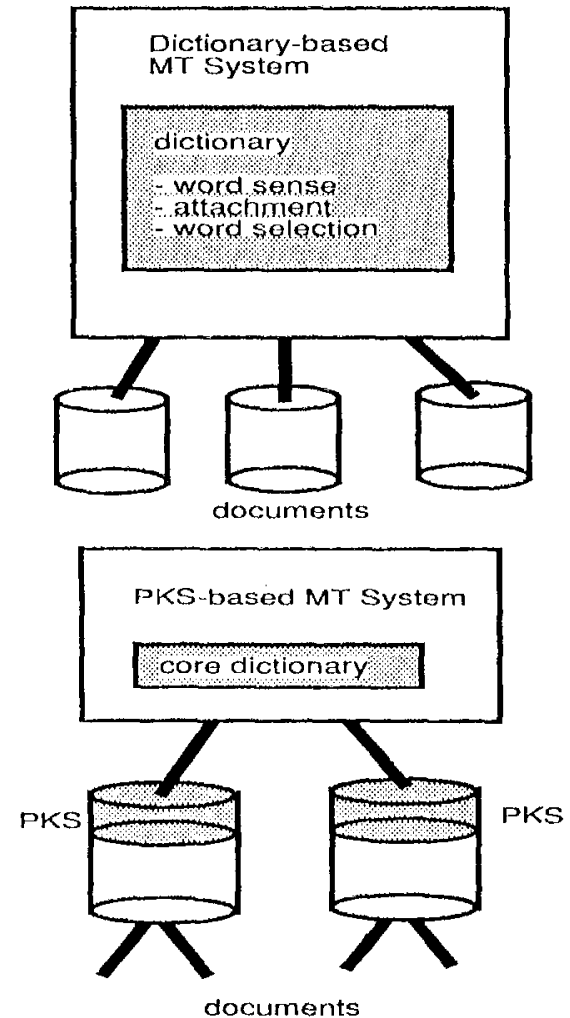

Figure 1: Jictionary-based and PKS-based MT Systems

in the previous section. When a new document is to be translated, the user either specifies a list of previously translated documents as a source of available PKSs, or lets the M'T system automatically choose them. Such a list of documents is called a document list.

Figure 1 compares a PKS-based MT system with that of a conventional diction ary-based MT system.

liven theogh in logical document may not be identified with a physical file, it is the easiest and most practical way to organize the hierarchy of documents. In practice, when translating technical documents, it is usual to translate the glossary first, agree on the translations of technical terms, and then work on individual chapters. This gives us a natural ordering of documents,

$$
\text { glossary } \rightarrow \text { chapter } 1 \rightarrow \text { chapter } 2 \ldots
$$

which is also used as an ordering of PKSs to be incorporated for machine translation. One way of automatically choosing the document $X$ for translating a new document $Y$ is to calculate the overlap of words contained in both $X$ and $Y$, and to find the $X$ with tho largest overlap. 'This idea is similar to the context identification method ${ }^{[13]}$, which is used effectively for word sense disambiguation. 
One important characteristic of this PKS organizittion is that it can be dynamically rearranged. We can invalidate some 1'KSs by simply removing a document from the document list, or validate a new set of P'KSs by adding its paired document to the list. This is extremely useful for domain-sensitive and context-sensitive translation, since a close look at documents in a seemingly similar domain will show that there are too many conflicting word senses and word selections to build a single consistent domain dictionary. ${ }^{2}$ In the worst case, the user has to keep on asking the system to prefer onc of several word senses as many times ats a new document arrives to be translated.

Another important observation is that the system can calculate the quality of preference information as follows:

1. Given a document list, find all the PKSs in the document list, and create a I'KS graph, which is a directed graph, for each type of PKS (see Figure 2):

- If the PISS is of type (PKL word sensej), create a node $N w_{i}$ for wordi, a nocto $N s_{j}$ for sense ${ }_{j}$, and a directed are $a_{i j}$ (labeled "sense") from $N w_{i}$ to $N s_{j}$.

- If the PKS is of type (PK2 wor $d_{i}$ role word $d_{j}$ ) create a node $N r_{i}$ for word $d_{i}$ a node $N l_{j}$ for word $_{j}$, and a directed arc $a_{i j}$ (laheled with at syntactic role) from $N r_{i}$ to $N l_{j}$.

- If the PKS is of type (PK3 word trans $_{j}$ ) create a node $N s_{i}$ for word $d_{i}$, a node $N t_{j}$ for trans, and it directed atc $a_{i j}$ (latrelod "trans") from $N s_{i}$ to $N t_{j}$.

2. Count the number $\mathrm{C} 1$ of conflicting arrs for the PK1 and PK3 graphs. That is, find the number of arcs leaving the same node but going to rlfferent nodes.

3. Count the number $\mathrm{C} 2$ of conflicting paths $\mathrm{s}^{3}$ for each pair of nodes $n 1$ and $n 2$, commected by an arc al in the PK2 graph, such that for some node n3, there are two arcs a 2 from $n 1$ to $n 3$, and a3 from $n 3$ to $n 2$, where 2 and a 3 have the sarne label (see Figure 3).

Intuitively, $\mathrm{Cl}$ shows a number of ambiguous word senses and word selections, and $\mathrm{C} 2$ shows possible attachment ambiguities in the given document list. ${ }^{4}$

\footnotetext{
"Recall the word senses of the word "line" in Section 2. Nll of them appear in the conmuter domain, notably in the areas of text editors, graphics, and hardware manuals, respectively. Our upproach nllows the user to adjust the sense dynumically for each type of manual.

${ }^{3} \mathrm{~A}$ path is a secquence of directed arcs. Conflicting pathe are two or more distinct sequences of pathe hetween an given pair of nodes.

'Suppose that $n 1$ is a prepositional phrase, $n 2$ is a verl, phrase, and $n 3$ is another prepositional phrage. Then, we lave an molsiguity in the $n 1$ attachment. Multiple ontgoing ares from an node in the PK2 graph do not necessarily imply armbiguities.
}

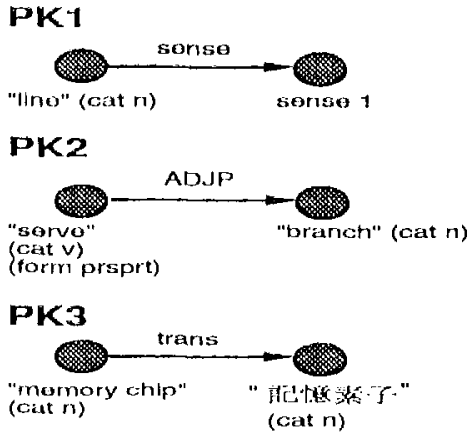

Figure 2: PKS graphs

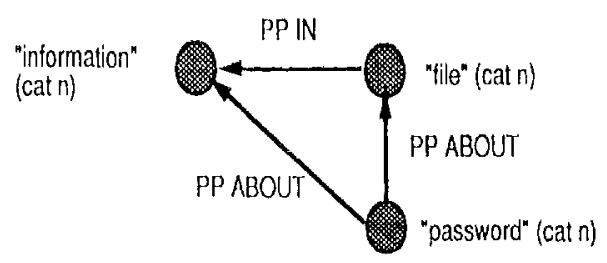

ligure 3: Conflicting paths in a. PKS graph

Therefore, a document, list wilh large C1 and/or C2 generally should be divided into smaller lists for consistent transation. In au ideal situation, C1 and C2 both should be 0 . Cale et al. ${ }^{[3]}$ and Nasukawa ${ }^{[7]}$ suggest that there is a strong tendency for C1 to be very small in a reasonable span of text.

It is easily shown that for any two document lists 1,1 and 12 (1, $\left.\cap \cap L_{2}=\phi\right)$, the numbers of conflicting ares and paths, $C_{11}, C_{12}, C_{21}$, and $C_{22}$, respectively, are monotonic. That is, the numbers C1 and C2 of conflicting arcs and paths of the combined document list $L_{1}(=\mathrm{I} 1 \cup \mathrm{L}, 2)$ satisfy $\mathrm{Cl} \geq C_{11}+C_{21}$ and $\mathrm{C} 2 \geq$ $C_{21}+C_{22}$.

\section{Disambiguation Method}

The basis of disambiguation of the three types of ambignity discussed in Section 2 is to prefer the last PKS in the document list that matches the ambiguity, and to interpret the PKS as a rule for selecting a word sense, phrasal attachment, or word transtation.

If there is no matching PKS, either the ambiguity is properly handled by the system, which results in no user correction in the documents, or it is new to the system. In the former case, the user will probably be satisfied with the translation by the system. In the latter case, the translation may have to be corrected by the user, but the interaction will be recorded as a new PKS and used for future disambiguation.

The matching algorithm for PK1 and PK3 rules is a simple exact matching of words and lexical features. If 
two or more PKS rules match the ambiguous word, the ages of the rules and the ordering of dlocuments in the document list uniquely determine the most preferable PKS. The PK2 rules, however, can be used with a more flexible matching algorithm ${ }^{[0,12]}$, since the coverage of PK2 rules would be very limited if two phrases (the modifier and modifiee phrases) had to match the rule exactly.

Once the document list has been given, the PK1 and PK3 rules can be polynomially converted into a simple lookup table, where the key is an ambiguous word, and only the most preferable rules are stored or retrieved. ${ }^{5}$ PK2 rules can be organized similarly as a ternary lookup table.

It should be noted that sentences in a document list can be utilized as an example base ${ }^{[9]}$, since the documents in the document list has already been transiated, and the translation of the source sentence is readily available. Indeed, the conventional matching algorithm for a flat example base has to be extended into in hierarchical one, where the latest translation has the highest priority, and PKSs must be equally taken into consideration.

\section{Knowledge Source Compilation}

When a set of documents in one domain grows considerably, or when the MT system is to be transported to a different environment, it is convenient to be able to compile PKSs into a single, portable user dictionary. The compilation is similar to the creation of lookup ta bles, described in the previous section. The numbers of conflicting arcs and paths should be carefully examined to see whether a given document list yields a consistent user dictionary. The user can rearrange the ordering of documents, and choose the most preferable among conflicting PKS rules to make the optimal user dictionary for the domain.

The rearrangement of documents in the document list does not change the resulting PKS graphs. It just. changes the preferences among the conflicting arcs or paths. Therefore, the optimal construction of a user dictionary does not have to consider an exponential number of possible document orderings, but only a polynomial number of the following pairwise constraints:

- If there are conflicting arcs $a_{1}, a_{2}, \ldots, a_{k}$ in tho PK1 (or PK3) graph, and the most preferable arc is $a_{i}$, the document $d_{i}$ having the PK1 (or PK3) rule for $a_{i}$ must be preceded by each document $d_{j}$ having the arc $a_{j}(j=1, \ldots, k, j \neq i)$.

- If there are conflicting paths $p_{1}, p_{2}, \ldots, p_{k}$ in the PK2 graph, and the most preferable path is $p_{i}$, the

${ }^{5}$ Alternatively, all the conflicting PKS rules cun be stored to give the user as many candidates as possible. clocument $d_{i}$ having the PK2 rule for the first arc in $p_{i}$ must be preceded by each document $d_{j}$ having the PK2 rule for the first are in $p_{j}(j=1, \ldots, k$. $j \neq i)$.

It is polynomially decidable whether there is an ordering of documents that satisfies all of the above constraints. An ordering of documents exists ifl the constraints are not cyclic (that is, iff there is a document $D$ that must precede itself). Fven if there is no linear ordering of such documents, the user dictionary can still be created from the user-selected arcs and paths. In this case, however, there is no natural correspondence between the user dictionary and a document list. Such a correspondence is indispensable if the user wishes to update the user dictionary when a new document is added at an arbitrary position in a document list. If the user dictionary is equivalently reducible to a document list, recompilation of the document list, into the user dictionary is straightforward. When no such equivalent list exists, a document may only be added to the tail of the list, thus overruling all the conflicting PKSS.

\section{Alternative Views of Knowledge Organization}

In Section 3, we took a simplified view of the PKS organization, which we may call an "optimistic organization." It was implicitly assumed that only the elements in PKSs can conflict with each other. However, the system's defanlt choice of word senses, may have satisfied a user, but may conflict with a PKS newly added to the document list. 'Ihus, PKSs need to be more carefully organized if the user thinks that the translation by the system is adequate without the PKSs. This view may be called a "pessimistic organization" of PKSs. The optimistic organization is easier to implement, while the pessimistic organization cau provide users with more consistent translation.

In the pessimistic organization, the conflicting know!edge has to be defined in terms of PKSs and the choices of word sense, phrasal attachment, and word translation by the system. 'Technically, it means that for every PKS in the document list, we have to examine if each rule in the PKS to determine whether it conflicts with a preceding PKS rule or a choice by the system. This is often very time-consuming. One way to deal with this pessimistic view is to keep track of the document list with which a new document is translated. It can easily be shown that time-consuming checking of PKS conflicts can be avoided by employing a monotonously growing sequence of document lists, $\left\{d_{1}\right\},\left\{d_{1}, d_{2}\right\}, \ldots,\left\{d_{1}, d_{2}, \ldots, d_{k}\right\}$ such that each doc11ment $d_{i}$ in the list has been translated by using the PKSs in the document list $\left\{d_{1}, d_{2}, \ldots, d_{i-1}\right\}$. 


\section{Conclusions}

In this paper, we have proposed a new machine translation mechanism based on portable knowledge sources. It provides MT' systems with an eflicient way of acquiring and utilizing the vital information from the user in order to gradually achieve correct translation in a multi-domain, multi-user enviromment. Since the document list (or a list of previously translated documents) is usually read-only, it is not only a convenimt unil for storing domain-oriented disambiguation knowledge, but also an ideal resource for machine trauslation that can be shared by many users.

We have started organizing our knowledge sources into a clocument list. The current documents consist of four IBM $A S / 400$ computer manuals with ahout, 22,000 sentences and a CA1) manual with about 10,000 sentences. Currently only about 1,200 sentences have been translated by using our prototypo MT system Shalt2 ${ }^{[1]}$, and corrected by the user for knowlerlge acquisition. The PKS rules are therefore few and not. ready for quantitative analysis. Some interesting occurrences of words, however, have already been found. In one of the above manuals, for example, the noun "part" was used in two different word senses: as a hard. ware component and as an abstract portion of a whole. The first usage of this noun, however, seemed more restricted: it was always modified by a proper noun. In one manual, the word "line" was clearly and consistently used to mean a specific row on the screen, while in another it was always used to mean a geomotric component.

The interaction of PKS rules has not been discussed in this paper, although it is a very interesting topic. Suppose that PK2 rules can also include the word senses for each word. It is not clear whether the word senses in the rules should be considered as conditional (valid only if the pair of modifier and modifiee is present.) or absolute (no matter what the modifier and modifice are). Only the latter case is handled by the J'Kl, and the former cases could be the exceptions to the P'K1 rules.

If the "one sense per document" assumption seems too strong, we can modify the P'K1 and PK3 rules by adding contextual dependency to the rules so that it word sense or a word translation is valid only if the word appears with a certain modifier or modifiee, hats at certain syntactic role, and so on.

\section{Acknowledgement}

I would like to thank Tetsuya Nasukawa and Naohiko Uramoto for many discussions which have gradually materialized into the idea of portable knowledge sources. The comments from Hideo Watanabe and lliroshi Nomiyama also contributed to the refinement of the technical contents. Michael McDopald, as usual, proofread the paper, and helped me write the final version.

\section{References}

1) C. Boitet and II. Blanchon. "Dialogue-Based MT for monolingual authors and the IIIDIA project". In Proce. of the Naltral Language P'rocessing P'acific Rim Sympo. sium 'D3, pages 208-222, Mukuoka, Japun, 1)ec. 1993.

2) $113 \mathrm{M}$ Corp. "IBM SAA AD/Cycle Translation Manager/2 Release 1.0". "Technical report, SII 12-5906, 1992.

3) W. Gale, K. Church, and D. Yarowsky. "One Sense Per Jiscourse". In Proc of the fith DARPA Speech and Natural language. Workshop, 1992.

1) II. Marnyama, II. Watanibe, and S. Ogino. "An In" teractive Japanese l'arser for Machine Translation". In P'roce of the 13 th International Conference on Compalalional Linguislics, pages 257-262, Helsinki, Aug. 1990.

5) S. W. Mclloy. "Using Multiple Knowledge Sourees for Word Sense Discrimination". Computalional Ligutistics, 18(1):1 30, Mar. 1992.

(6) K. Nagaio. "Dependency Analyzer: A Knowledge-Based Approach to Structural Disambiguation". In Proce of the 13th International Conference on Computational Linguistics, panges 282-287, Helsinki, Aug. 1990.

7) 'T. Nusukawa. "Discource Constraint in Computer Manuals". In Iroc. of the 5th International Conference on Theoretical and Methodological Issues in Machine Translation, pages 183-194, Kyoto, Japaal, July 1993.

8) J. Pustegovsky. "Ihe Generative Lexicon". Compulalional Linguistics, 17(4):109 441, December 1991.

9) S. Sato and M. Nagato. "l'oward Memory-based Translittion". In Proe. of the t3ih International Conference on Computational Linguistics pages 247-252, Helsinki, A.ug. 1090.

10) S. Sumita and IT. Iida. "Experiments and Prospects of Fxample-Based Machine 'Iranslation". In Proce of the 29th Arnual Meeting of the Association for Computational Linguistics, pages 185-192, Berkeley, June 1991.

11) K. Takeda, N. Uramoto, T. Nasukawa, and T. 'I'sutsumi. "Shalt2 - A Symmetric Machine 'Tanslation System with Conceptuial 'transfer". In Proc. of the lith International Conference on Compulational Linguistics, pages $1034-1038$, July 1992.

12) N. Uramoto. "Lexical and Structural Disambiguation Using an lixample-Batse". In Prot. of the End JapanAustralia Joint Symposium on NII, pages 150-160, Oct. 1991.

13) D. Yarowsky. "Word-Sense Disambiguation Using StaListical Models of Roget's Cutegories 'Irained on Large Corpora". In Proce of the Iflih International Conference on Computational Linguistics, pages 454-460, July 1992 